\title{
Chemical Reaction Theory: Summarizing Remarks
}

\author{
Donald G. Truhlar \\ Department of Chemistry and Supercomputer Institute, University of Minnesota, \\ Minneapolis, MN 55455-0431, USA
}

\section{Introduction}

Faraday Discussions often revisit a topic in new forms at periodic intervals. The present discussion is in many ways a successor to several earlier conferences on gas-phase dynamics:

Title

Inelastic Collisions of Atoms and Simple Molecules

Molecular Dynamics of Chemical Reactions of Gases

Molecular Beam Scattering

Potential Energy Surfaces

Dynamics of Elementary Gas-Phase Reactions

Structure and Dynamics of Reactive Transition States

$\begin{array}{cc}\text { Number } & \text { Year } \\ 33 & 1962 \\ 44 & 1967 \\ 55 & 1973 \\ 62 & 1977 \\ 84 & 1987 \\ 91 & 1991\end{array}$

There is also some overlap with Discussion 67 (1979) on State-Selected Kinetics and Discussion 75 (1983) on Intramolecular Kinetics. However, the present symposium differs from all these in two very important ways: (1) condensed-phase reactions are also included, and (2) the discussion is focused exclusively on theory. With these aspects in mind one could include a number of earlier predecessors as well, for example the famous 1937 discussion mentioned in Miller's Spiers Memorial Lecture, the 1954 discussion on fast reactions, and the 1982 discussion on electron and proton transfer. The reader who compares selected papers from these past conferences to papers from the present conference will note a dramatic improvement in the reliability and sophistication of theoretical models, especially as applied to complex systems.

A critical organizing element in planning a theoretical treatment of any chemical reaction is the division into potential energy surfaces and dynamics. In my summary remarks on the present Discussion, I will consider both of these problems. Furthermore, in organizing the remarks on dynamics I have focused on three themes in the papers presented here: dynamical bottlenecks and reaction coordinates, dynamics of nonadiabatic processes, and theoretical progress in gas-surface dynamics spurred by experimental advances.

\section{Potential energy surfaces}

A potential energy surface (PES, also called a force field) is a prerequisite for a dynamics calculation. At the same time, we use dynamics calculations to learn more about potential energy surfaces. Thus there exists a symbiotic relationship:

$$
\left\{\begin{array}{c}
\text { potential energy } \\
\text { surfaces }
\end{array}\right\} \rightleftharpoons\left\{\begin{array}{c}
\text { dynamics } \\
\text { calculations }
\end{array}\right\}
$$


An unfortunate theme of this conference was difficulties with potential energy surfaces. For example:

(1) The 'complete lack of agreement between theory and experiment' for $\mathrm{F}+\mathrm{H}_{2}$ at a collision energy of $1.35 \mathrm{kcal}$ results from an incorrect $\mathrm{HF}+\mathrm{H}$ limit on the StarkWerner potential surface. ${ }^{1}$

(2) 'To our surprise we found very strange oscillatory $N_{i}(E) \ldots$ These resonanceslike structures... are due to a deep well.... Since it is believed that the well is very likely unphysical....'2

(3) 'Quantum mechanical calculations are not in good agreement with experiment. This suggests that the PES might not permit enough tunneling [the barrier is too thick] and/or that the zero point energies at the transition state are too large.'

(4) 'The previous calculations were based on the previous version of the PES, with an artificial well.... The effect previously attributed to the azimuthal anisotropy of the PES at the bridge site geometry was in fact due to the artificial well. ${ }^{3}$

(5) 'An important point for the present FEP calculation is how well the force field reproduces the hydrogen bonding pattern .... The HF/6-31G* results are well reproduced with the exception of the TS complex with the hydrogen bond at N1, which is too weakly bound by $1.9[!] \mathrm{kcal}^{.4}$

(6) 'If classical force fields are to be used in $\mathrm{MO}+\mathrm{MM}$ methods, e.g., for efficient optimization, then some reparametrization . . . may be necessary. ${ }^{5}$

A survey of the various discussion papers showed a variety of types of potential energy surfaces were used; these are summarized in Table 1 . We see a prevalence of analytic potential energy surfaces for gas-phase studies, density functional theory (DFT)

Table 1 Which types of potential energy surfaces (PESs) were used in the discussion papers?

\begin{tabular}{|c|c|c|c|}
\hline & gas & $\begin{array}{l}\text { gas/solid } \\
\text { interface }\end{array}$ & liquid \\
\hline \multicolumn{4}{|l|}{ electronically adiabatic } \\
\hline \multicolumn{4}{|l|}{ for reaction-path properties, transition states } \\
\hline $\mathrm{MM}^{a}$ (molecular mechanics) & 0 & 1 & 1 \\
\hline $\mathrm{PM}^{b}+\mathrm{MM}$ (coupled) & 0 & 0 & 1 \\
\hline DFT & 0 & 2 & 0 \\
\hline DFT + MM (uncoupled) & 0 & 0 & 1 \\
\hline $\mathrm{CCSDT} / \mathrm{pDZ}: \mathrm{MP} 2 / \mathrm{p} 5 Z^{c}$ & 1 & 0 & 0 \\
\hline \multicolumn{4}{|l|}{ for dynamics } \\
\hline previously fitted PESs & 4 & 0 & 0 \\
\hline PES fitted to DFT & 0 & 3 & 0 \\
\hline \multicolumn{4}{|l|}{ for direct dynamics } \\
\hline BPW91 $/ 6-31 \mathrm{G}(\mathrm{d}, \mathrm{p})(9$ atoms, 3 trajectories $)$ & 1 & 0 & 0 \\
\hline \multicolumn{4}{|l|}{$\begin{array}{l}\text { electronically non-adiabatic } \\
\text { for dynamics }\end{array}$} \\
\hline CASPT $2^{e} / 6-31 \mathrm{G}(\mathrm{d}, \mathrm{p})$ & 1 & 0 & 0 \\
\hline previously fitted PESs & 1 & 0 & 0 \\
\hline matrix MM & 0 & 0 & 1 \\
\hline \multicolumn{4}{|l|}{ for direct dynamics } \\
\hline 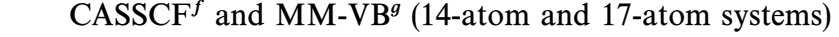 & 1 & 0 & 0 \\
\hline
\end{tabular}

a 'MM' is a 'previously fitted PES' with general parameters rather than system-specific parameters. ${ }^{b}$ Parametrized Model 3 (Stewart's parametrization of neglect-of-diatomic-differentialoverlap molecular orbital theory). ${ }^{c}$ Following Morokuma, the colon separates levels in a duallevel or multi-level calculation. ${ }^{d}$ Becke-Perdew-Wang-'91 version of DFT, which employs Becke's 1988 non-local exchange functional and the PW91 non-local correlation functional. ${ }^{e}$ Second order perturbation theory with a CASSCF reference state. ${ }^{f}$ Complete-active-space self-consistent-field theory. ${ }^{g} \mathrm{MM}$-valence bond. 
for gas/solid interfaces involving metals, ${ }^{3,6-9}$ and molecular mechanics (MM) for liquidphase kinetics, as well as for reactions at the surface of solid ice. Direct dynamics, in which electronic structure calculations are performed 'on the fly' as required by the dynamics algorithm, was used for only a few applications in this Discussion, perhaps due to the large errors that may be incurred ${ }^{10,11}$ in the most popular ${ }^{12}$ direct dynamics algorithms, which are purely classical for the nuclear motion.

I would like to single out for special attention the multi-level focal point scheme in the paper by King et al. ${ }^{13}$ This scheme ${ }^{13,14}$ is compared to some previous ${ }^{15-34}$ duallevel and multi-level schemes for electronic structure calculations in Table 2. As indicated there, the focal point scheme combines desirable features of several other schemes; this makes it an attractive and powerful method. For example, like G2 theory, it separates the convergence with respect to level of electron correlation from convergence with respect to one-electron basis set; but, unlike G2 theory, it attempts to estimate the converged answers, not just to carry out a particular high-level calculation in a computationally efficient manner.

An example showing how the method is applied in practice is the calculation of the ${ }^{3} \mathrm{~A}^{\prime \prime}$ ketene dissociation barrier. ${ }^{13}$ The effect on this barrier of double substitutions in the Hartree-Fock reference wavefunction is $2300 \mathrm{~cm}^{-1}$; this effect was estimated by extrapolation from a sequence of basis sets up to cc-pV5Z. The effect of single substitutions, of a higher-order treatment of doubles coupled to singles, and of a perturbation treatment of connected triple substitutions is $702 \mathrm{~cm}^{-1}$; the sum of these effects was calculated by extrapolating from calculations with cc-pVDZ, cc-pVTZ and cc-pVDZ/TZ basis sets, which are smaller than cc-pV5Z. Finally the effect of going from uncoupled to coupled triple substitutions is estimated to be $62 \mathrm{~cm}^{-1}$ based on calculations with a cc-pVDZ basis set. Thus we see that larger (low-order) electron correlation effects were estimated with large basis sets and smaller (higher-order) electron correlation effects were estimated with smaller basis sets. The resulting accuracy of the ${ }^{3} \mathrm{~A}^{\prime \prime}$ ketene dissociation barrier is estimated as $\lesssim 200 \mathrm{~cm}^{-1}$, and perhaps it is even more accurate (by up to a factor of 2 - see discussion remarks).

The progress in recent years in using electronic structure calculations to provide potential energy surfaces has been dramatic. The state of the art at the time of the above mentioned 1937 Faraday Discussion was the semiempirical London-Eyring-Polanyi valence bond method. By 1967, there was a recognition that $a b$ initio methods could contribute, and in his 1967 summarizing remarks, Polanyi said, 'Not one of the papers

Table 2 Quantum electronic structure: dual-level and multi-level methods

\begin{tabular}{|c|c|c|}
\hline methods & reference & notes \\
\hline // methods & popularized by GAUSSIAN package ${ }^{15}$ & \\
\hline G2 theory & Curtiss, Raghavachari, Trucks, Pople ${ }^{16}$ & $a$ \\
\hline CBS & Petersson, Radom, and co-workers ${ }^{17-19}$ & $b$ \\
\hline SEC & Brown, Truhlar ${ }^{20}$ & $b$ \\
\hline SAC & Gordon, Truhlar and co-workers ${ }^{21,22}$ & $b$ \\
\hline PCI-X (same as SAC) & Siegbahn, Blomberg, Svensson ${ }^{23,24}$ & $b$ \\
\hline IMOMO & Humbel, Sieber, Svensson, Morokuma ${ }^{25,26}$ & \\
\hline IMOMO(G2MS) & Froese et $a ._{.}^{27}$ & $a, c$ \\
\hline ONIOM & Svensson et al. ${ }^{28}$ & $c$ \\
\hline IMOHC & Corchado, Truhlar ${ }^{22,29}$ & \\
\hline cc-pV(X, $\left.X^{\prime}\right) Z$ & Dunning, Feller, Martin, Taylor, . & \\
\hline focal point scheme & East, Allen, ${ }^{14}$ King, Allen, Ma, Schaefer ${ }^{13}$ & $a, b, c$ \\
\hline
\end{tabular}

${ }^{a}$ These methods separate 1-particle (basis set) and $n$-particle (correlation level) convergence. ${ }^{b}$ These methods aim to extrapolate to complete CI limit (experimental accuracy). ${ }^{c}$ These methods involve more than 2 levels. 
contributed to the present Discussion dealt with the a priori calculations of potential energy surfaces .... Our greatest lack at the present time is a manageable theory of the potential energy surfaces. For $\mathrm{H}_{3}^{+}$and $\mathrm{H}_{3}$ a computation of 'chemical accuracy' is certainly possible today. For systems with more than 3 atoms, we may expect calculations of chemical accuracy over the next five years or so. ${ }^{35}$ This was true, and already in 1968 , Shavitt ${ }^{36}$ estimated the $\mathrm{H}_{3}$ barrier height to be $9.8 \mathrm{kcal}$ based on a combination of electronic structure calculations and empirical comparison to experiment, and this value is within $0.2 \mathrm{kcal}$ of the currently accepted value. However, ab initio potential surfaces were slower then expected to make their appearance at Faraday discussions, and in 1973 summarizing remarks Polanyi reported, ${ }^{37}$ 'I can report that $a b$ initio surfaces have not figured significantly, and I am surprised.' The wait was ended at a 1977 discussion where an $a b$ initio surface was presented for a reactive system, $\mathrm{NH}_{2}{ }^{+}$, based on configuration interaction with single and double substitutions and a polarized double zeta oneelectron basis set. ${ }^{38}$ By 1998, we take it for granted that $a b$ initio calculations can give reliable answers, at least for gas-phase problems. The situation can be summarized by the Dirac-style quotation: 'The equations can be written down, but their solutions are hard.' In the condensed phase, though, the situation is much more unsettled.

Here are some condensed-phase electronic structure challenges that have not been met.

(1) Carry out a converged calculation of the solvation energy of the $\mathrm{Na}^{+}$ion in water at $298 \mathrm{~K}$.

(2) Carry out a converged calculation of the solution-phase geometry and solvation energy of $\mathrm{CH}_{3} \mathrm{OH}$. (We would also like to ask for the solution-phase dipole moment of $\mathrm{CH}_{3} \mathrm{OH}$ or even its average net charge, but these are not well defined.)

(3) Carry out a converged calculation of the free energy of activation of any solutionphase reaction.

One could argue that we still don't have 'converged' calculations for, say, $\mathrm{NH}_{2}{ }^{+}$or ketene in the gas phase, but the nature of the situation is quite different. For $\mathrm{NH}_{2}{ }^{+}$or ketene, we are very confident that we have the tools, and that it is simply a matter of including higher levels of electron correlation and larger one-electron basis sets, i.e., a focal point scheme can converge the results to whatever accuracy we desire, given a fast computer, a good bankroll, and some patience. For solution-phase work, though, we still have no consensus on what method to use. How can one systematically converge the correlation energy between solute and solvent electrons for a macroscopic solvent? Since $c a$. 1990, many methods for electronic structure in solution have become available, mainly self-consistent reaction field methods. How reliable are they? We do not have converged benchmark results such as are available for testing gas-phase electronic structure methods, for example, Pekeris' converged calculations for $\mathrm{He}$ or Kolos and Wolniewicz's converged calculations for $\mathrm{H}_{2}$. (Even gas-phase dynamics has its converged benchmarks, e.g., the gas-phase barrier height of $\mathrm{H}_{3}$ is known to $0.01 \mathrm{kcal}$, and gasphase quantum dynamics for $\mathrm{D}+\mathrm{H}_{2} \rightleftharpoons \mathrm{HD}+\mathrm{H}$ has been well converged.) Solutionphase electronic structure does not even have the forerunners of these benchmarks, such as Hylleraas's calculations on gas-phase He or James and Coolidge's calculations on gas-phase $\mathrm{H}_{2}$. Ultimately, in the gas phase, our tests on small systems like $\mathrm{OH}+\mathrm{H}_{2}$ or $\mathrm{CN}+\mathrm{H}_{2}$ give us confidence in the reliability or unreliability of our methods when we tackle larger systems like $\mathrm{OH}+\mathrm{C}_{3} \mathrm{H}_{8}$ or organic cycloaddition reactions. Until one has such benchmarks for the liquid phase, it will be hard to test the reliability of liquidphase dynamics methods such as those used in the paper by Repasky and Jorgensen. ${ }^{4}$ One might ask: is condensed-phase electronic structure not only waiting for its Hylleraas, but even waiting for its Schrödinger? This is not clear. The Schrödinger formulation of quantum mechanics seems to be the most convenient way to treat systems of interacting fermions, but the Feynman path integral formulation of quantum mechanics may be the best approach for applying quantum mechanics to systems where thermal 
fluctuations are an intrinsic part of the problem. Perhaps a combination of Schrödinger and Feynman methods is called for.

Given the above situation we still cannot answer the following question: Do ab initio methods have advantages over semiempirical methods (e.g., molecular mechanics with polarization terms or hybrid DFT with empirical parameters) for liquid-phase dynamics?

Similar questions arise at gas/solid interfaces: can we believe the results of large-scale DFT calculations? One of the stated goals of the paper by McCormack et al. ${ }^{3}$ is to test DFT in this context by carrying out accurate dynamics calculations for a close fit to an extensive set of DFT calculations and comparing the results to experiment, which is the tried-and-true testing procedure for PESs in the absence of converged electronic structure benchmarks. However, for gas/solid interfaces, one can also envision more systematic applications of conventional electronic structure methods such as those pioneered by Whitten. ${ }^{39}$

In addition to this general difficulty, there are other, more specific difficulties in obtaining condensed-phase force fields for dynamics. For example, Burton et al. ${ }^{5} \mathrm{com}-$ mented that 'A ... problem here is the protonation state of the phosphate since $\mathrm{HPO}_{4}{ }^{2-}$ could be a better nucleophile [than $\mathrm{H}_{2} \mathrm{PO}_{4}{ }^{-}$.' This raises two interesting issues. First, protonation states of phosphate and of amino acid side chains are often unknown. The $\mathrm{p} K_{\mathrm{a}}$ in the enzyme may differ significantly from the aqueous value, and of course it differs enormously from the gas-phase value. Second, $\mathrm{HPO}_{4}{ }^{2-}$ is presumably unstable in the gas phase, and we cannot use the comfortable strategy of first converging the calculation in the gas phase and then solvating. Related examples are kinetics problems that are intrinsically condensed-phase in nature, e.g., proton relays ${ }^{40,41}$ and condensed-phase reaction paths involving solvent motion. ${ }^{42,43}$

\section{Dynamics}

Which types of dynamical methods were used in the discussion papers? Table 3 gives a summary. We see that accurate quantum mechanics was used in half the gas-phase cases but in only one ${ }^{3}$ condensed-phase study, and that case still involves a rigid metal lattice, which is a serious approximation. In the liquid phase, an approximate quantal calculation was reported for a system with an impressively large 11286 degrees of freedom. ${ }^{44}$

Table 3 Which types of dynamical methods were used in the discussion papers?

\begin{tabular}{lccc}
\hline & gas & $\begin{array}{c}\text { gas/solid } \\
\text { interface }\end{array}$ & liquid \\
\hline reaction path properties (TST ${ }^{a}$ data) & 1 & 2 & 3 \\
statistical model (TST rate constant) & 0 & 0 & 1 \\
statistical + diffusion or friction & 0 & 0 & 0 \\
trajectories - classical, quasiclassical & 1 & 1 & 1 \\
statistical + tunneling & 0 & 0 & 0 \\
statistical + hopping & 1 & 0 & 0 \\
QM/CM (quantum mechanics/classical mechanics) & 2 & 1 & 0 \\
approx. QM-time-dependent & 1 & 1 & $1^{b}$ \\
Accurate QM-TI (time-independent) & 2 & 0 & 0 \\
TD (time-dependent) & 3 & $1^{c}$ & 0 \\
TI/TD & 1 & 0 & 0 \\
\hline
\end{tabular}

${ }^{a}$ Transition state theory. ${ }^{b}$ Ben-Nun et $a l^{44}$ (11286 degrees of freedom).

${ }^{c}$ McCormack et al. ${ }^{3}$ (6 degrees of freedom). 


\subsection{Dynamical bottlenecks and reaction coordinates}

I noted three major themes in the area of dynamical bottlenecks and reaction coordinates: (i) how to find them in complex systems, (ii) how to use them for rearrangement scattering, and (iii) their role in electronically non-adiabatic processes.

Several papers were concerned with dynamical bottlenecks, reaction coordinates, and reaction paths in complex systems.

Gertner and Hynes ${ }^{42}$ made effective use of a collective solvent coordinate for a charge transfer reaction that is defined as the energy difference, for a given solvent configuration, between the system with the charge localized on the donor and the system with the charge localized on the acceptor. This idea has been used fruitfully by Marcus, Warshel, and Hynes and their collaborators ${ }^{45-47}$ in previous studies. The method is particularly useful when solvent effects are dominated by electrostatic interactions between the solute and many solvent molecules.

Hammer ${ }^{9}$ applied the Ulitsky-Elber algorithm ${ }^{48}$ for refining the reaction path to the problem of dissociation of NO at a Pd surface. For a stepped surface he calculated a 46 kcal barrier when the surface is relaxed along the reaction path as compared to a $52 \mathrm{kcal}$ barrier when the surface is held rigid (static, unrelaxed) at its bulk geometry. This very large difference provides a dramatic example of the importance of allowing all the degrees of freedom to participate in the reaction coordinate.

Sprik $^{43}$ presented a new method for sampling reaction paths in liquids when the reaction coordinate involves the first solvation shell of the solvent. This method allows the use of collective reaction paths for umbrella sampling, and it should be useful for calculating the free energy of activation for complex catalytic reactions such as enzyme catalysis and acid-base catalysis and reactions with solvent as a coordinating ligand, which is a common situation for organometallics.

Bolhuis et al. ${ }^{49}$ presented a method for sampling condensed-phase reaction paths when one has no preconceived notions of transition mechanisms or transition states. The new method was illustrated by application to a system with 14 degrees of freedom. They also emphasized a point of view by which the transition state is not a point but rather a distribution of points. They did not compare this approach to the widely used definition $^{50-56}$ of a transition state as a variationally determined hypersurface separating reactants from points; both approaches treat the transition state as a collection of points, not as a single point, and they allow the treatment of systems with parallel saddle points.

The three papers just mentioned ${ }^{9,43,49}$ are all examples of renewed interest in 'chain of states' methods ${ }^{48,57-68}$ for finding reaction paths, saddle points, free energy of activation maxima, and dynamical bottlenecks. In these methods a reaction path is found by optimizing the elements of a chain between reactants and products rather than by steepest descent from a transition state or steepest ascent from a single minimum. The calculation simultaneously yields the reaction path and the transition state. As dynamicists tackle harder and more complex problems, there is a growing realization that this kind of algorithm has important practical advantages over quasi-Newton methods ${ }^{69}$ for finding the transition state of the particular reaction under consideration. It is interesting to note that some of the chain methods are related to the variational formulation of classical dynamics. ${ }^{70}$ Chain methods can also be used in conjunction with a quasiNewton or steepest ascents method in a two-stage algorithm. ${ }^{71}$

Another paper whose goal is the determination of reaction paths is Goldfield's. ${ }^{72}$ She used quantal wavepackets to show that a double torsional reaction path is more likely than a single torsion for stereomutation of cyclopropane.

Although the above papers are all very different in detail, taken together they illustrate that great progress is being made in finding reaction paths, reaction coordinates, and dynamical bottlenecks for complex systems. A related area of research involves the 
choice of coordinates for quantum mechanical scattering calculations on chemical reactions and the use of appropriate coordinates to exploit the simplifications made possible in such reactions by recognizing the existence of a dynamical bottleneck.

One of the most fundamental problems in chemical reaction theory is that the most appropriate coordinates for reactants are not the same as the most appropriate coordinates for products. A very old solution to this problem is to define a reaction coordinate that measures progress along a reaction path and to define a complete set of other coordinates, called reaction-path coordinates, orthogonal to this. ${ }^{73}$ In the present Discussion, Hammes-Schiffer ${ }^{40}$ proposed using reaction-path coordinates (without reactionpath curvature) as a way to simplify the time propagation in studies of gas-phase reactions.

Another way to try to simplify dynamical calculations on chemical reactions is to center attention on the region around the dynamical bottleneck or on times close to that at which the system passes through the dynamical bottleneck. For example, starting trajectories at the transition state, which is a form of rare-event sampling, has a long history, and it has been used in both purely classical ${ }^{52,74-81}$ and semiclassical ${ }^{82-85}$ implementations. The quantal analog is initializing a wavepacket at the transition state. Light and Zhang $^{2}$ presented a refined version of this idea for calculating exact quantal rate constants with the minimum number of wavepacket propagations allowed by variational transition state theory (by starting wavepackets at the variational transition state where the integrated density of states is a minimum ${ }^{86}$ ). A technical aspect handled nicely in this paper is defining a manageable, but globally satisfactory reaction coordinate that divides reactants from products for a four-body rearrangement.

Peng et $a .^{87}$ also build on an old idea, namely to write the wavefunction for a rearrangement as

$$
\begin{aligned}
\Psi= & \Psi_{1} \text { (expressed in coordinate system of reactants) } \\
& +\Psi_{2} \text { (expressed in coordinate system of products) }
\end{aligned}
$$

In early work this led to coupled equations for $\Psi_{1}$ and $\Psi_{2}$ which could be solved as integrodifferential equations ${ }^{88}$ or by linear algebraic variational methods. ${ }^{89}$ Peng et al. refine this idea by using source and sink optical potentials to decouple $\Psi_{1}$ and $\Psi_{2}$ in either a time-independent or time-dependent formalism. This allows them to calculate accurate quantal rate constants with the minimum number of reactant-to-product coordinate transformations. Althorpe et al. ${ }^{90}$ presented a further refinement of the placement of the optical potentials that allows greater computational efficiency. Optionally one can add $\Psi_{3}$ expressed in coordinates appropriate to the dynamical bottleneck region, ${ }^{87}$ also an idea which was used previously without optical potentials. ${ }^{88,89,91}$

A subtheme running through the above work is the development of methods for calculating accurate quantal rate constants without calculating unnecessary details, e.g., without calculating the scattering matrix first. ${ }^{2,87,92}$ For example, the very elegant Light-Zhang wavepacket method discussed above appears to represent a pinnacle in efficient calculations of reaction rates without state-selective detail. For larger systems one can use this kind of a formalism as a starting point for approximations. ${ }^{93} \mathrm{We}$ can hope that this approach will eventually become practical for systems with many more degrees of freedom. A promising note along these lines was a poster ${ }^{94}$ presenting converged wavepacket calculations for a system with 24 degrees of freedom 'in a few hours'. Even though the method is not completely general, this kind of result is encouraging for future progress. The simple algorithm of Balint-Kurti et al., ${ }^{95}$ involving a single recursion relation in real arithmetic, illustrates another direction of progress in making wavepacket propagation more practical for complex systems.

Although none of the discussion papers employed variational transition state theory with a transmission coefficient based on semiclassical multidimensional tunneling 
calculations ${ }^{53,56,96}$ (see Table 3), that combination of methods was represented in two poster papers (one on $\mathrm{H}+\mathrm{C}_{2} \mathrm{H}_{4}{ }^{97}$ and one on $\mathrm{OH}+\mathrm{H}_{2} \mathrm{O}{ }^{98}$ ), and it remains the best available method for calculating thermal rate constants for complex systems. A strength of this approach is that it requires a minimum of electronic structure information (potential energy surface information) because it requires only enough information to identify and characterize the valleys around the dynamical bottleneck and terrain around the dominant tunneling paths.

Concluding the summary of work on the theme of reaction coordinates and dynamical bottlenecks, we note two papers in this area that are concerned with electronically non-adiabatic processes. ${ }^{99,100}$ These are discussed in the next section.

\subsection{Electronically non-adiabatic dynamics}

Another major theme at the present Discussion is that significant progress has been made in the treatment of electronically non-adiabatic reactions.

Tully ${ }^{101}$ presented a new derivation of trajectory surface hopping (TSH) methods and concluded that TSH in the adiabatic representation is superior to TSH in a diabatic representation or to the Ehrenfest time-dependent self-consistent-field method. Discussion following this paper further elucidated the successes and deficiencies of these methods.

Garavelli et al. ${ }^{100}$ presented TSH calculations for a 14-atom system by direct dynamics calculations. They also emphasized that a lot can be learned without full dynamics calculations. For example, they studied the coupling of vibrational energy redistribution to the reaction coordinate (in the same 14-atom system) by calculating the 'initial relaxation direction' (IRD), which they defined as the minimum energy path (MEP) from a Franck-Condon point. Thus IRD joins the well known IRC as another special case of MEP, and the MEP is shown to be a powerful tool for ultrafast photochemistry.

A second paper concerned with ultrafast photochemistry is that by Ben-Nun et al. ${ }^{44}$ Ben-Nun et al. presented calculations by the full multiple spawning method. In principle this method converges to exact quantum mechanics as the number of basis functions increases if they are fully coupled. Here the method is used with 16 nuclear basis states, each spawning $c a .10$ more. Each basis state is a product of 3 Gaussians per atom (one for each Cartesian coordinate); for the 3762-atom system considered this yields 11286 Gaussians. The 16 basis states were propagated independently of each other, i.e., uncoupled. It is possible to obtain reasonably accurate results with uncoupled frozen Gaussians, but the probabilities degrade with increasing integration time. ${ }^{102}$ Nevertheless the method is appealing because of its computational efficiency. It is, however, more work than TSH. Is it more accurate? Martinez pointed out that the method has worked well on every problem on which it has been tested, but it has been tested on different problems ${ }^{103}$ from those which have used TSH. It would be interesting to define a standard version of the method (e.g., precisely three spawned basis functions per each transversal of a non-adiabatic region, with a universal algorithm for spawning them) and test it widely. Another point worth making about this paper is that the authors employed matrix molecular mechanics for the coupled potential energy surfaces, and progress at modeling electronically excited potentials currently lags our ability to model groundstate surfaces.

The final subject to be discussed in the area of electronically non-adiabatic processes is the subject of non-adiabatic dynamics induced by spin-orbit coupling. ${ }^{99,104}$

Schatz et al. ${ }^{104}$ studied the abstraction of an $\mathrm{H}$ atom from $\mathrm{HCl}$ by both $\mathrm{Cl}$ and $\mathrm{Cl}^{*}$, where $\mathrm{Cl}^{*}$ denotes the spin-orbit-excited state. The products are $\mathrm{HCl}$ and either $\mathrm{Cl}$ or $\mathrm{Cl}^{*}$. What fraction of the thermal rate constant is due to $\mathrm{Cl}+\mathrm{HCl} \rightleftharpoons \mathrm{HCl}+\mathrm{Cl}$, as opposed to reactions involving $\mathrm{Cl}^{*}$ as reactant and/or product, e.g., $\mathrm{Cl}^{*}+\mathrm{HCl} \rightleftharpoons \mathrm{HCl}$ 
$+\mathrm{Cl}$ ? The answer they found is $98 \%$ at $220 \mathrm{~K}$ and $90 \%$ at $1000 \mathrm{~K}$. But if they artificially decrease the spin-orbit splitting from 2.5 to $0.25 \mathrm{kcal}$, the answer becomes $\leqslant 33 \%$ at all temperatures, indicating a much greater role for electronically non-adiabatic reaction. The discussion of this paper brought out the fact that electronic angular momentum is treated in the 'pure precession limit'. There are no more complete calculations available to show whether this approximation is valid. Thus we await further work for a completely definitive treatment of the role of electronic angular momentum in this reaction.

Morokuma et al..$^{99}$ presented an extension of transition state theory (TST) for the treatment of non-adiabatic processes induced by spin-orbit coupling. As noted by the authors, the treatment is similar in some respects to previous ${ }^{105-109}$ statistical models for electronically non-adiabatic reactions. The process considered by Morokuma et al..$^{99}$ is illustrated in Fig. 1. There are three dynamical bottlenecks on the way from reactants to products, but the central one, involving intersystem crossing from the doublet manifold to the singlet one, is the controlling bottleneck. Morokuma et al. treat the intersystem crossing by non-adiabatic TST. In adiabatic TST one writes

$$
k^{\mathrm{TST}}(T) \propto\langle\delta(s) \dot{s} \theta(\dot{s})\rangle
$$

where $T$ is temperature, $s$ is the reaction coordinate, $\delta$ is the Dirac delta function, $\theta$ is a Heaviside function, and an overdot denotes a time derivative, whereas in non-adiabatic TST one writes

$$
k_{\text {quench }}(T) \propto\langle\delta(s) \dot{s} \theta(\dot{s})\rangle \frac{\left\langle\delta(s) \dot{s} \theta(\dot{s}) P_{\text {quench }}(\boldsymbol{x})\right\rangle}{\langle\delta(s) \dot{s} \theta(\dot{s})\rangle}
$$

where $P_{\text {quench }}(x)$ is the local quenching probability at local geometry $x$. Thus the rate constant is a TST rate constant times a statistically averaged quenching probability. In the application by Morokuma et al., $P_{\text {quench }}(x)$ is approximated by a one-dimensional model that yields $c a \cdot 10^{-4}$, and the resulting $k_{\text {quench }}(T)$ is less than $10^{-2}$ of the experimental value. One possible fix is to scale down the frequencies to make a wider, more accessible bottleneck region, and another is to scale up the spin-orbit coupling that causes the quenching. As noted by the authors, neither of these is a very satisfactory fix. The conclusion is that the one-dimensional model is probably inadequate, and a multidimensional $P_{\text {quench }}(x)$ should be used. This is not entirely surprising since onedimensional models are known to be unreliable for non-adiabatic collisions. ${ }^{110}$ In my

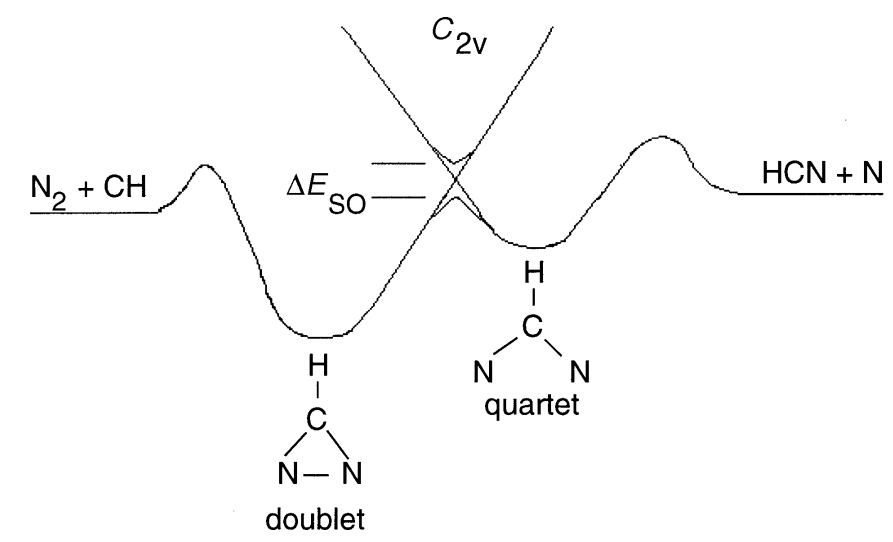

Fig. 1 Schematic potential energy diagram for $\mathrm{N}_{2}+\mathrm{CH}\left({ }^{2} \Pi\right) \rightleftharpoons \mathrm{HCN}+\mathrm{N}\left({ }^{4} \mathrm{~S}\right)$ by way of a doublet saddle point, followed by intersystem crossing followed by a quartet saddle point. The spin-orbit splitting at a $C_{2 \mathrm{v}}$ non-adiabatic transition state is drawn larger than scale. 
opinion, the details of this study should not overshadow the kind of vistas that it opens. Instead of thinking about intersystem crossing in the traditional way as a delocalized event governed in an uncertain way by the shapes of the relevant potential energy surfaces and couplings, this kind of treatment allows us to focus on more specific and more localized features of the potential energy surfaces and to better understand the multidimensional nature of the problem by elucidating the role of features like the width of the dynamical bottleneck region.

\subsection{Gas/surface interface dynamics}

A theme that runs through the papers on dynamics at gas/surface interfaces is that theoretical progress is spurred by exciting advances in experiments.

The Skinner Prize for best student poster paper was awarded to G. Boentgen for a presentation $^{111}$ on photochemical isotope effects that epitomizes this theme. The stimulus for this paper was a scanning tunneling microscope (STM) experiment by Avouris and co-workers ${ }^{112}$ showing that when provided with an energy in excess of $6 \mathrm{eV}$, hydrogen atoms chemisorbed on a reconstructed $\mathrm{Si}(100)$ surface are 60 times more likely to be desorbed than deuterium atoms. Applying a combination of theoretical methods, Boentgen showed that the result is explainable if the energy deposited in the $\mathrm{Si}-\mathrm{H}$ antibonding orbital returns to the surface in less than one femtosecond.

Another goad to theory is the use of STM to identify the microscopic site of a surface-catalyzed dissociation reaction. For example, Zambelli et al. ${ }^{113}$ used scanning tunneling microscopy to observe a stepped $\mathrm{Ru}(0001)$ surface after dissociation of $\mathrm{NO}$ at $315 \mathrm{~K}$. They observed a diffusion profile of $\mathrm{N}$ atoms in the vicinity of the step-on both sides of the step. The $\mathrm{O}$ atoms (which diffuse faster than $\mathrm{N}$ on the surface) were found in islands, somewhat farther from the step - again on both sides. They concluded that dissociation occurs at the step, which follows directly from the observed diffusion profile. More speculatively, they proposed that dissociation occurs at the frontmost atom of the top step. (One must be careful here not to make the mistake of assuming that it is harder to diffuse across the step than toward it or away from it or that it is harder to diffuse up a step rather than down it; diffusion across a step in either direction may actually be easier than diffusion on terraces. ${ }^{114}$ ) A possible explanation ${ }^{113}$ for dissociation at the frontmost atom of the top step is that low-coordinated $\mathrm{Ru}$ atoms have a narrower $\mathrm{d}$ band and consequently exhibit more $\mathrm{d}$ backbonding to the $\pi^{*}$ orbital of NO.

This kind of experiment is a spur to theoretical efforts to understand the site dependence of catalytic reactions. In response, Hammer ${ }^{9}$ presented a detailed electronic structure study of the competition between dissociation and desorption for nitric oxide on Pd on the (111) terrace and on the stepped (211) surface. He used DFT (with the PerdewWang 1991 density functional). On (111) he found identical barrier heights of $52 \mathrm{kcal}$ for dissociative chemisorption and desorption of NO. In such a case one would expect to observe the entropically favored desorption process. On (211) the barrier for dissociative chemisorption decreases to $46 \mathrm{kcal}$ and that for desorption increases to $55 \mathrm{kcal}$, so dissociation should occur mainly at the step. Thus DFT provides useful insight into the best site for catalysis.

Understanding the role of promoters and poisons has long been a goal of theoretical chemists studying heterogeneous catalysis: 'The addition of minor elements, sometimes referred to as promoters, is a common occurrence in the industrial practice of heterogeneous catalysis. The exact role of such promoters is not always clear. ${ }^{115}$ This sentiment is also echoed by others: 'The term promoter is applied to substances that are not by themselves catalytically active but which allow the active phase to function to its maximum capacity. Their mode of action is not always clearly understood. ${ }^{116}$ At the present discussion, the question was taken up by Bird: 'The rate of many surface reactions is profoundly influenced by the presence of co-adsorbed species. Despite the great 
practical importance of such promoters and poisons in industrial catalysis their role is not well understood at a fundamental level. ${ }^{8}$ Bird studied the dissociation of $\mathrm{H}_{2}$ on a $\mathrm{Cu}(111)$ surface in the presence of a coadsorbed oxygen atom. He carried out DFT electronic structure calculations with the Perdew-Wang-'91 density functional, and he examined five possible bridge sites where dissociation may occur, as illustrated in Fig. 2. In the absence of coadsorbed $\mathrm{O}$, the barrier height $V^{\ddagger}$ was calculated to be $12.7 \mathrm{kcal}$. The barrier heights at the five sites near $\mathrm{O}$ are given in Table 4. One observes both poisoning $\left(V^{\ddagger}>12.7 \mathrm{kcal}\right)$ and promotion $\left(V^{\ddagger}<12.7 \mathrm{kcal}\right)$. The explanation varies with the site. Poisoning at site 2 is blamed on direct interaction with O. Poisoning at site 3 is explained by the theory of Hammer and Nørskov. ${ }^{117}$ In particular, in this theory, the interaction energy with the surface of the transition state for the dissociating $\mathrm{H}_{2}$ is stabilized by interaction of the antibonding orbital of $\mathrm{H}_{2}$ with the metal $\mathrm{d}$ band; this lowers the energy of the transition state. However the $\mathrm{Cu}$ atoms labeled $\mathrm{Cu} 1$ in Fig. 2 have their $\mathrm{d}$ orbitals stabilized by about $1 \mathrm{eV}$ by $\mathrm{O}$. This weakens their interaction with the $\sigma^{*}$ orbital of $\mathrm{H}_{2}$, partially poisoning the catalytic effect. Interestingly, the

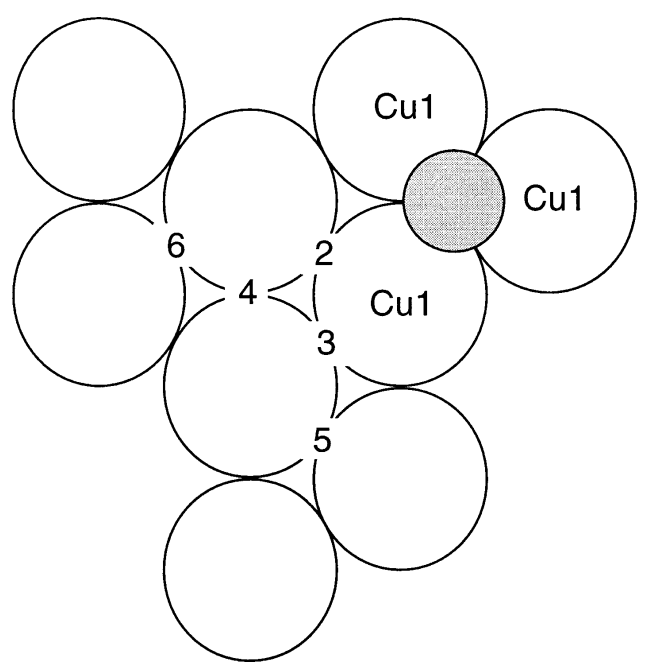

Fig. 2 Schematic diagram of an oxygen atom (shaded) on the (100) surface of $\mathrm{Cu}$. The three nearest $\mathrm{Cu}$ atoms are labeled $\mathrm{Cu} 1$, and five possible bridge sites where dissociation of $\mathrm{H}_{2}$ may occur are labeled 2 through 6 .

Table 4 Barrier heights for dissociation of $\mathrm{H}_{2}$ on $\mathrm{Cu}(111)$ in the presence of oxygen coadsorbate

\begin{tabular}{ccc}
\hline site & nearest $\mathrm{Cu}$ atoms & \\
\hline$b$ & $\mathrm{U}-\mathrm{U}$ & 12.7 \\
2 & $1-\mathrm{kcal}$ & 27.1 \\
3 & $1-\mathrm{U}$ & 16.7 \\
4 & $\mathrm{U}-\mathrm{U}$ & 13.1 \\
5 & $\mathrm{U}-\mathrm{U}$ & 11.4 \\
6 & $\mathrm{U}-\mathrm{U}$ & 11.5 \\
\hline
\end{tabular}

${ }^{a} 1$ denotes a $\mathrm{Cu}$ neighboring $\mathrm{O}$ (these atoms are labeled $\mathrm{Cu} 1$ in Fig. 2); $\mathrm{U}$ denotes any other (unperturbed) $\mathrm{Cu}$.

${ }^{b}$ Bare surface. 
Hammer-Nørskov theory does not explain the weaker effects at sites 4-6. Nor can these effects be explained by long-range electrostatics, which are estimated to affect the barrier by only $c a$. $0.3-0.4 \mathrm{kcal}$. (The effect is small because $\mathrm{O}$ is adsorbed very close to the metal which screens the electrostatics; the effect may be 1-2 kcal for alkalis. ${ }^{8}$ ) Bird eventually explained the promotion effect of $>1 \mathrm{kcal}$ at sites 5 and 6 as due (perhaps) to less repulsion by the surface. The effect at site 4 is too small to blame on any one effect. The impressively detailed analysis of various qualitative explanations of the poisoning and promoting effects at the various sites illustrates the level of microscopic detail that is now possible for understanding the chemical kinetics of catalytic reactions.

Another stimulating experiment is the work of Hou et al. ${ }^{118}$ on desorption of molecular $\mathrm{D}_{2}$ from $\mathrm{Cu}(111)$ at $925 \mathrm{~K}$. They used time-of-flight measurements to determine the translational energy of desorbed $\mathrm{D}_{2}$ (in the range $E_{\text {trans }}=0.3-0.8 \mathrm{eV}$ ) and state-resolved resonance-enhanced multiphoton ionization to determine its rotational quantum number $j$. The latter measurements were carried out as a function of the polarization angle of the incident radiation, yielding information about $K$ (the projection quantum number of $j$ ) and hence about the rotational alignment of the desorbing $\mathrm{D}_{2}$. Lowvelocity $\mathrm{D}_{2}$, which escapes by tunneling, was found primarily in the helicopter alignment, whereas high-velocity $\mathrm{D}_{2}$, escaping by an overbarrier process, showed a mixture of helicopter and cartwheel alignments. The six-dimensional wavepacket calculation of McCormack et al. ${ }^{3}$ yielded a cross-section ratio $\sigma_{\text {helicopter }} / \sigma_{\text {cartwheel }}$ of less than 1 for $E_{\text {trans }}<0.45 \mathrm{eV}, c a .2$ for $E_{\text {trans }}=0.55 \mathrm{eV}$ and $c a .3$ for $E_{\text {trans }}>0.7-0.9 \mathrm{eV}$. The more relevant quantity for comparison with experiment is the quadrupole alignment for which there is some qualitative agreement with experiment. Another such comparison is provided by the recent work of Dai and Light ${ }^{119}$ (see the discussion remarks by Light) who, for rotational states $j \geqslant 6$, found excellent agreement with experiment not only for the rotational alignment but for the dependence on $E_{\text {trans }}$. Shalashilin et al. ${ }^{7}$ pointed out that the PES used by Dai and Light is very close to their new potential energy surface based on DFT calculations (which was successful for rotational distributions of the products of Eley-Rideal reactions but not for vibrational distributions but which has a barrier that may be too high by $\sim 0.2 \mathrm{eV}$ ). McCormack and Kroes pointed out that their own PES has considerably more azimuthal corrugation than the PES used by Dai and Light, and that the strong corrugation in angular directions (azimuthally and polarly) in their minimum barrier to reaction is what results in the lack of preference seen for helicopter reaction at low collision energies for rotational state $j=4$, which apparently agrees with experiment better than the low-j results of Dai and Light. It is quite possible that neither surface is accurate enough to predict the experimental alignment correctly for all $j$. McCormack and Kroes pointed out that it is important to distinguish carefully between the anisotropy of the DFT calculations and the anisotropy of the PES fitted to the DFT calculations. Relating the dynamics results more specifically to the PES features is sure to be an interesting problem for further work. ${ }^{3}$

\section{Conclusion}

The pre-meeting announcement stated: 'Predictions of the dynamics of small molecules can now be made as reliable as measurements, and the accuracy of calculations on more complicated problems (organic reactions, reactions at surfaces and in solution) is improving at a very fast pace.' The papers presented here demonstrated that these statements are indeed true. Furthermore, even when the calculations are not quantitatively accurate, the theoretical treatment can be very useful. For example, in such a case it is stimulating to ask why a calculation isn't accurate. In other cases observing what happens in the simulations stimulates qualitative thinking. This Discussion has shown that a variety of types of calculations can lead to improved understanding of dynamical processes, and they can aid us in developing qualitative concepts to guide our thinking. 
The Discussion was very well organized. On behalf of all the attendees, I would like to express our thanks to the organizing committee, David Clary (Chairman), Jonathan Connor, Ian Hillier, Steve Holloway, W. C. Mackrodt, David Manolopoulos and Mike Robb, for arranging a very stimulating discussion.

\section{References}

1 J. F. Castillo and D. E. Manolopoulos, Faraday Discuss., 1998, 110, 119.

2 J. C. Light and D. H. Zhang, Faraday Discuss., 1998, 110, 105.

3 D. A. McCormack, G-J. Kroes, E-J. Baerends and R. C. Mowrey, Faraday Discuss., 1998, $110,267$.

4 M. P. Repasky and W. J. Jorgensen, Faraday Discuss., 1998, 110, 379.

5 N. A. Burton, M. J. Harrison, J. C. Hart, I. H. Hillier and D. W. Sheppard, Faraday Discuss., 1998, 110, 463

6 G. R. Darling and S. Holloway, Faraday Discuss., 1998, 110, 253.

7 D. Shalashilin, B. Jackson and M. Persson, Faraday Discuss., 1998, 110, 287.

8 D. M. Bird, Faraday Discuss., 1998, 110, 335.

9 B. Hammer, Faraday Discuss., 1998, 110, 323.

10 F. T. Smith, Discuss. Faraday Soc., 1962, 33, 293.

11 J. Espinosa-García, J. C. Corchado and D. G. Truhlar, J. Am. Chem. Soc., 1997, 119, 9891.

12 M. Parrinello, in Modern Techniques in Computational Chemistry: MOTECC-19, ed. E. Clementi, ESCOM, Leiden, 1991, p. 833.

13 R. A. King, W. D. Allen, B. Ma and H. F. Schaefer III, Faraday Discuss., 1998, 110, 23.

14 A. L. L. East and W. D. Allen, J. Chem. Phys., 1993, 99, 4638.

15 W. J. Hehre, L. Radom, P. v. R. Schleyer and J. A. Pople, Ab Initio Molecular Orbital Theory, Wiley, New York, 1986.

16 L. A. Curtiss, K. Raghavachari, G. W. Trucks and J. A. Pople, J. Chem. Phys., 1991, 94, 7221.

17 M. R. Nyden and G. A. Petersson, J. Chem. Phys., 1981, 75, 1843.

18 J. W. Ochterski, G. A. Petersson and J. A. Montgomery, Jr., J. Chem. Phys., 1996, 104, 2598.

19 M. W. Wong and L. Radom, J. Phys. Chem. A, 1998, 102, 2237.

20 F. B. Brown and D. G. Truhlar, Chem. Phys. Lett., 1985, 117, 307.

21 M. S. Gordon and D. G. Truhlar, J. Am. Chem. Soc., 1986, 108, 5412.

22 J. C. Corchado and D. G. Truhlar, in Combined Quantum Mechanical and Molecular Mechanical Methods, ed. J. Gao and M. A. Thompson, American Chemical Society, Washington, in press.

23 P. E. M. Siegbahn, M. R. A. Blomberg and M. Svensson, Chem. Phys. Lett., 1994, 223, 35.

24 P. E. M. Siegbahn and R. H. Crabtree, J. Am. Chem. Soc., 1996, 118, 4442.

25 S. Humbel, S. Sieber and K. Morokuma, J. Chem. Phys., 1996, 105, 1959.

26 M. Svensson, S. Humbel and K. Morokuma, J. Chem. Phys., 1996, 105, 3654.

27 R. D. J. Froese, S. Humbel, M. Svensson and K. Morokuma, J. Phys. Chem. A, 1997, $101,227$.

28 M. Svensson, S. Humbel, R. D. J. Froese, T. Matsubara, S. Sieber and K. Morokuma, J. Phys. Chem., 1996, 100, 19375.

29 J. C. Corchado and D. G. Truhlar, J. Phys. Chem., 1998, 102, 1895.

30 A. K. Wilson and T. H. Dunning, Jr., J. Chem. Phys., 1997, 106, 8718.

31 D. A. Dixon, D. Feller and K. A. Peterson, J. Phys. Chem. A, 1997, 101, 9405.

32 J. M. L. Martin and P. R. Taylor, J. Chem. Phys., 1997, 106, 8620.

33 C. W. Bauschlicher, Jr. and A. Ricca, J. Phys. Chem. A, 1998, 102, 4722.

34 D. G. Truhlar, Chem. Phys. Lett., in press.

35 J. C. Polanyi, Discuss. Faraday Soc., 1967, 44, 293.

36 I. Shavitt, J. Chem. Phys., 1968, 49, 4048.

37 J. C. Polanyi, Faraday Discuss. Chem. Soc., 1973, 55, 389.

38 C. F. Bender, J. H. Meadows and H. F. Schaefer III, Faraday Discuss. Chem. Soc., 1977, 62, 59.

39 J. Whitten, NATO ASI Ser. B, 1992, 283, 375

40 S. Hammes-Schiffer, Faraday Discuss., 1998, 110, 391.

41 M. T. Nguyen, T. Raspoet and L. G. Vanquickenborne, Faraday Discussion 110, poster presentation.

42 B. J. Gertner and J. T. Hynes, Faraday Discuss., 1998, 110, 301.

43 M. Sprik, Faraday Discuss., 1998, 110, 437.

44 M. Ben-Nun, F. Molnar, H. Liu, J. C. Phillips, T. J. Martinez and K. Schulten, Faraday Discuss., 1998, 110, 447.

45 R. A. Marcus, J. Chem. Phys., 1956, 24, 966.

46 A. Warshel, J. Phys. Chem., 1982, 86, 2218.

47 J. T. Hynes, in Solvent Effects and Chemical Reactivity, ed. O. Tapia and J. Bertrán, Kluwer, Dordrecht, 1996, p. 231.

48 A. Ulitzky and R. Elber, J. Chem. Phys., 1990, 92, 1510.

49 P. G. Bolhuis, C. Dellago and D. Chandler, Faraday Discuss., 1998, 110, 421. 
50 E. Wigner, J. Chem. Phys., 1937, 5, 720.

51 J. Horiuti, Bull. Chem. Soc. Jpn, 1938, 13, 210.

52 J. C. Keck, Discuss. Faraday Soc., 1962, 33, 173.

53 S. C. Tucker and D. G. Truhlar, in New Theoretical Concepts for Understanding Organic Reactions, ed. J. Bertrán and I. G. Csizmadia, Kluwer, Dordrecht, 1989, p. 291.

54 B. C. Garrett and G. K. Schenter, Int. Rev. Phys. Chem., 1994, 13, 263.

55 G. C. Schenter, G. Mills and H. Jónsson, J. Chem. Phys., 1994, 101, 8694.

56 D. G. Truhlar, B. C. Garrett and S. J. Klippenstein, J. Phys. Chem., 1996, 100, 12771.

57 T. A. Halgren and W. N. Lipscomb, Chem. Phys. Lett., 1977, 49, 225.

58 A. Jensen, Theor. Chim. Acta, 1983, 63, 269.

59 L. R. Pratt, J. Chem. Phys., 1986, 85, 5045.

60 R. C. Boehm and L. L. Lohr, J. Phys. Chem., 1989, 93, 3430.

61 C. Choi and R. Elber, J. Chem. Phys., 1991, 94, 751.

62 D. A. Liotard, Int. J. Quantum Chem., 1992, 44, 723.

63 E. M. Sevick, A. T. Bell and D. W. Theodorou, J. Chem. Phys., 1993, 98, 3196.

64 G. Mills, H. Jónsson and G. K. Schenter, Surf. Sci., 1995, 324, 305.

65 J. J. Mortensen, Y. Morikawa, B. Hammer and J. K. Nørskov, J. Catal., 1997, 169, 85

66 M. Mavrikakis, L. B. Hansen, J. J. Mortensen, B. Hammer and J. K. Nørskov, in Transition State Modeling for Catalysis, ed. D. G. Truhlar and K. Morokuma, American Chemical Society, Washington, in press.

67 E. Sandré, M. C. Payne, I. Stich and J. D. Gale, in Transition State Modeling for Catalysis, ed. D. G. Truhlar and K. Morokuma, American Chemical Society, Washington, in press.

68 H. Jónsson, G. Mills and K. W. Jacobsen, in Classical and Quantum Dynamics in Condensed Phase Simulations, ed. B. J. Berne, G. Ciccotti and D. F. Coker, to be published.

69 M. L. McKee and M. Page, Rev. Comput. Chem., 1993, 4, 35.

70 R. E. Gillilan and K. R. Wilson, J. Chem. Phys., 1992, 97, 1757.

71 C. Peng and H. B. Schlegel, Isr. J. Chem., 1994, 33, 449.

72 E. Goldfield, Faraday Discuss., 1998, 110, 185.

73 M. A. Eliason and J. O. Hirschfelder, J. Chem. Phys., 1959, 30, 1426.

74 J. B. Anderson, J. Chem. Phys., 1973, 58, 4684.

75 C. H. Bennett, in Diffusion in Solids, ed. A. S. Nowick and J. J. Burton, Academic Press, San Francisco, 1975, p. 73 .

76 J. A. Montgomery, D. Chandler and B. J. Berne, J. Chem. Phys., 1979, 70, 4056

77 J. A. McCammon and M. Karplus, Annu. Rev. Phys. Chem., 1980, 31, 29.

78 A. F. Voter and J. D. Doll, J. Chem. Phys., 1985, 82, 80.

79 B. J. Gertner, K. R. Wilson and J. T. Hynes, J. Phys. Chem., 1989, 90, 3537.

80 J. B. Anderson, Adv. Chem. Phys., 1995, 91, 381.

81 M. Strnad, M. T. C. Martins-Costa, C. Millot, I. Tuñon, M. F. Ruiz-Lopez and J. L. Rivail, J. Chem. Phys., 1977, 106, 3643.

82 I. W. Smith, J. Chem. Soc., Faraday Trans. 2, 1981, 77, 747.

83 J. W. Tromp and W. H. Miller, Faraday Discuss. Chem. Soc., 1987, 84, 441.

84 D. G. Truhlar and B. C. Garrett, Faraday Discuss. Chem. Soc., 1987, 84, 464.

85 M. Ferrario, D. Laria, G. Ciccotti and R. Kapral, J. Mol. Liq., 1994, 61, 37.

86 B. C. Garrett and D. G. Truhlar, J. Chem. Phys., 1979, 70, 1593.

87 T. Peng, W. Zhu, D. Wang and J. Z. H. Zhang, Faraday Discuss., 1998, 110, 159.

88 W. H. Miller, J. Chem. Phys., 1969, 50, 407.

89 D. G. Truhlar, J. Abdallah, Jr. and R. L. Smith, Adv. Chem. Phys., 1974, 25, 211.

90 S. C. Althorpe, D. J. Kouri and D. K. Hoffmann, Faraday Discussion 110, poster presentation.

91 G. J. Tawa, S. L. Mielke, D. G. Truhlar and D. W. Schwenke, Adv. Mol. Vib. Collision Dyn. B, 1994, 2, 45.

92 W. H. Miller, Faraday Discuss., 1998, 110, 1.

93 U. Manthe, Faraday Discussion 110, poster presentation.

94 H-D. Meyer, A. Jackle and M. C. Heitz, Faraday Discussion 110, poster presentation.

95 G. G. Balint-Kurti, A. I. Gonzalez, E. M. Goldfield and S. K. Gray, Faraday Discuss., 1998, 110, 169.

96 T. C. Allison and D. G. Truhlar, in Modern Methods for Multidimensional Dynamics Computations in Chemistry, World Scientific, Singapore, 1998, p. 618.

97 J. Villà, A. González-Lafont, J. M. Lluch, J. C. Corchado and D. G. Truhlar, Faraday Discussion 110, poster presentation.

98 I. H. Williams, M. R. Hand, C. F. Rodriguez and G. G. Balint-Kurti, Faraday Discussion 110, poster presentation.

99 K. Morokuma, Q. Cui and Z. Liu, Faraday Discuss., 1998, 110, 71.

100 M. Garavelli, F. Bernardi, M. Olivucci, T. Vreven, S. Klein, P. Celani and M. A. Robb, Faraday Discuss., 1998, 110, 51

101 J. C. Tully, Faraday Discuss., 1998, 110, 407. 
102 R. T.Skodje and D. G. Truhlar, J. Chem. Phys., 1984, 80, 3123.

103 M. Ben-Nun and T. J. Martinez, J. Chem. Phys., 1998, 108, 7244.

104 G. C. Schatz, P. McCabe and J. N. L. Connor, Faraday Discuss., 1998, 110, 139.

105 J. C. Tully, J. Chem. Phys., 1974, 61, 61.

106 G. E. Zahr, R. K. Preston and W. H. Miller, J. Chem. Phys., 1975, 62, 1127.

107 M. Desouter-Lecomte, C. Sannen and J. C. Lorquet, J. Chem. Phys., 1983, 79, 894.

108 J. C. Lorquet and B. Leyh-Nihant, J. Phys. Chem., 1988, 92, 4778.

109 M. S. Topaler and D. G. Truhlar, J. Chem. Phys., 1997, 107, 392.

110 S. L. Mielke, G. J. Tawa, D. G. Truhlar and D. W. Schwenke, Chem. Phys. Lett., 1995, $234,57$.

111 G. Böndgen and P. Saalfrank, Faraday Discussion 110, poster presentation.

112 P. Avouris, R. E. Walkup, A. R. Rossi, T-C. Shen, G. C. Abeln, J. R. Tucker and J. W. Lyding, Chem. Phys. Lett., 1996, 257, 148.

113 T. Zambelli, J. Wintterlin, J. Trost and G. Ertl, Science, 1996, 273, 1688.

114 T. N. Truong and D. G. Truhlar, J. Chem. Phys., 1990, 93, 2125.

115 M. W. Roberts and C. S. McKee, in Chemistry of the Metal-Gas Interface, Oxford University Press, Oxford, 1978, p. 541

116 G. C. Bond, in Heterogeneous Catalysis, Oxford University Press, Oxford, 1987, p. 541

117 B. Hammer and J. K. Nørskov, Surf. Sci., 1995, 343, 211; 1996, 359, 306(E).

118 H. Hou, S. J. Gulding, C. T. Rettner, A. M. Wodtke and D. J. Auerbach, Science, 1997, 277, 80.

119 J. Dai and J. C. Light, J. Chem. Phys., 1998, 108, 7816. 